\title{
Review: ROI Based Medical Image Compression using DCT and HAAR Wavelet
}

\author{
Sarvarinder Singh ${ }^{1}$, Er. Sarabjit Singh ${ }^{2}$ \\ M.Tech Research Student, Guru Kashi University, Talwandi Sabo ${ }^{1}$ \\ Assistant Professor, Guru Kashi University, Talwandi Sabo ${ }^{2}$
}

\begin{abstract}
Image compression addresses the problem of reducing the amount of data required to represent a digital image. There are some areas of medicine where it is sufficient to maintain high image quality only for diagnostically significant regions, for example, tumor section of the brain MRI. This paper represents a compression method based on ROI and its performance analysis focusing on Discrete Cosine Transform technique. It is used for reducing the redundancy that is nothing but avoiding the replica data. The DCT is a mathematical function that transforms digital image data from the spatial domain to the frequency domain.
\end{abstract}

Keywords: Images, lossy compression, Medical image compression, Region of Interest, Discrete Cosine Transform.

\section{INTRODUCTION}

An image is essentially a 2-D signal processed by the human visual system. The signal representing the image usually is a analog form. Medical imaging has a great impact on the diagnosis of diseases and surgical planning. Image compression is an function of data compression that encodes the original image with few bits. The objective of image compression is to diminish irrelevance and redundancy of the image data in order to be able to store or transmit data in an proficient form. Image compression means the reduction of the size of the image data, while retraining necessary information.

\section{A. Image Compression}

Image compression addresses the difficulty of reducing the quantity of data required to characterize a digital image. Through image compression the image is represented in small storage space, in this manner the storage/transmission requirements of the image are also reduced [1]. Image compression is achieved by the removal of one or more of the three basic data redundancies and these redundancies are:

\footnotetext{
Coding Redundancy

$>$ Inter pixel redundancy

$>$ Psycho visual redundancy
}

Coding redundancy is present when less than optimal code words are used. It consists in using variable length code words selected as to match the statistics of the original source. This type of coding is always reversible and usually implemented using lookup tables [1]. An interpixel redundancy is from correlations between the pixels of an image because in image neighboring pixels are not statistically independent. This is due to the relationship between the adjacent pixels of an image. This kind of redundancy is called Inter-pixel redundancy or spatial redundancy. Psycho visual redundancy is due to data that is ignored by the human visual system (i.e. visually non essential information).
Most of the times, the psycho physical aspects of human vision have confirmed that the human eye does not respond with equal sensitivity to all incoming visual information. A few piece of information are more necessary than others.

\section{B. Image compression techniques}

The image compression techniques are generally classified into two categories depending whether or not an exact replica of the original image could be reconstructed using the compressed image.

These techniques are:-

Lossless technique

Lossy technique

These both techniques are explained as follows:

Lossless compression technique In lossless compression techniques, the original image can be completely recovered from the compressed (encoded) image [8]. These are also called noiseless since they do not add noise to the signal (image). It is known as entropy coding since it uses statistics/decomposition techniques to eliminate/minimize redundancy. Lossless compression is used only for a few applications with strict necessities such as medical imaging. Following techniques are incorporated in lossless compression:

$>$ Run length encoding

$>$ Huffman encoding

$>$ LZW coding

$>$ Area coding

Lossy compression technique Lossy schemes provide much higher compression ratios than lossless schemes [8]. By this scheme, the decompressed image is not identical to the original image, but reasonably close to it and is used in many applications. In lossy methods, a little information is lost as the high compression ratio is the main objective [3]. 


\section{DIGITAL IMAGE}

A digital image may be defined as a two-dimensional functional $f(x, y)$, where $x$ and $y$ are spatial coordinates, $f$ is the amplitude of any pair of coordinate of $\mathrm{x}, \mathrm{y}$ that is called the intensity or gray level of the image at that point. Digital images are electronic snapshots scanned from documents, for instance photographs, manuscripts, printed texts and artwork. A digital image is a numeric representation of a two-dimensional image. Digital images may be of raster type or vector type. Raster type digital images are composed of pixels (picture elements), mean pixels are stored in computer memory as a raster image or raster map, a two-dimensional array of small integers. Each pixel is assigned a total value such as black, white, grey level or color, which is represented in binary code zeros and ones, at a single point in the image. Pixels are a little like grain particles in a conventional photographic image, but arranged in a regular pattern of rows and columns and store information somewhat differently. The binary digits (bits) for each pixel are stored in a sequence by a computer and often reduced to a mathematical representation (compressed). The bits are then interpreted and read by the computer to produce an analogy version for display or printing. When using digital equipment to capture, store, modify and view photographic images, they must first be converted to a set of numbers in a process called digitization or scanning. Computers are very good at storing and manipulating numbers, so once your image has been digitized you can use your computer to archive, examine, alter, display, transmit, or print your photographs in an incredible variety of ways. Vector type images resulted from mathematical vector. In terms, a vector consists of point that has both direction and length. The density of pixels in an image is referred to as its resolution. The higher the resolution, the more information the image contains.

\subsection{Types of Digital Images}

For photographic reason, there are two important types of digital images such as color and black and white images. Color images are made up of colored pixels while black and white images are made up of gray shade pixels.

\subsubsection{Grayscale Images}

Grayscale image is classically made up of pixels represented by 8 bits per pixel (bpp) each of which holds a single number corresponding to the gray level of the image at a particular location. These gray levels span the full range from 0 (black) to 255 (white) in a series of very fine steps, normally 256 different gray colors. Since the eye can barely distinguish about 200 different gray levels

\subsubsection{Color Images}

A digital color image is stored as three dimensional arrays and uses 24 bits ( 3 bytes) to represent each pixel. Each pixel holds three numbers corresponding to the red, green, and blue levels of the image at a particular location. Red, green, and blue colors component scale lies between 0 to 255, also known as RGB format. RGB are the primary colors for mixing light, these also called additive primary colors. Any color can be created by mixing the correct amounts of red, green, and blue light. This corresponds to roughly 16.7 million different possible colors. Note that for images of the same size, a black and white version will use three times less memory than a color version.

\subsubsection{Binary Images}

Binary images use only a single bit to represent each pixel. Since a bit can only exist in two states like on or off, every pixel in a binary image must be one of two colors, usually black or white. This inability to represent intermediate shades of gray is what limits their usefulness in dealing with photographic images.

\subsubsection{Indexed Color Images}

Some color images are created using a limited palette of colors, typically 256 different colors. These images are referred to as indexed color images because the data for each pixel consists of a palette index indicating which of the colors in the palette applies to that pixel. There are several problems with using indexed color to represent photographic images. First, if the image contains more different colors than are in the palette, techniques such as dithering must be applied to represent the missing colors and this degrades the image. Second, combining two indexed color images that use different palettes also create problems because of the limited number of available colors.

\section{MEDICAL IMAGING}

Digital Image Processing is one of the most popular research areas from last four decades, in the whole world. Reason for this popularity is huge variety of implementations of Digital Images in almost each era of technology. Medical Imaging is one of the major application areas of Digital Image Processing. Various medical diagnosing techniques are using digital images of human body as the deciding factors for next medical treatment [6]. Imaging in Medicine is being used to image the human body since 1960. Imaging helps a lot to represent the internal problem of body in visual manner. Medical Sonography is an ultrasound-based diagnostic medical imaging procedure used to visualize muscles, tendons, and lots of internal organs, their volume, structure and any pathological lesions with valid time tomographic images.

Imaging techniques extensively used in medicine include X-Ray, Magnetic Resonance Imaging (MRI), Ultrasound and Computed Tomography (CT). This technique is being used on large scale in field of neurology, cardiology, gynecology, urology and other medical diagnostic systems [7]. 3D MRI contains multiple slices containing parts of body, requires all information of that part. The compression method for 3D medical image includes the properties like: high lossless compression ratios, resolution scalability, quality scalability etc [11]. Current compression schemes produce high compression rates if 
loss of quality is inexpensive. However, in most cases physicians may not afford any insufficiency in diagnostically important regions of images; called ROIs. An approach that brings a high compression rate with good quality in the ROI is thus necessary. [5].

\section{REGION OF INTEREST IN MEDICAL IMAGING}

The ROI is important in medical applications where certain parts of the image are of higher diagnostic significance than others. All regions of medical image do not have equal importance as only some portions are diagnostically important. In such cases, these regions require to be encoded at a higher superiority than the background, lesser important regions [4]. In medical imaging data loss in the diagnostically important regions (ROI) is not affordable. The ROI can be determined from a DICOM image and the rest of image can be considered as Non-ROI of an image. The general theme is to preserve quality in diagnostically critical regions, while allowing lossy compression of the other regions. During the image transmission for telemedicine purposes, ROIs are required to be transmitted first or at the high priority [11]. In the ECG (Heartbeat) signals, ROI mask is used to separate the signal region from the non-signal region in the image that is to be transformed [10].

\section{PROPOSED METHOD}

The Discrete Cosine Transform (DCT) is a mathematical transformation technique that is used to change a spatial representation of data into a frequency symbol. A data in the frequency domain contains the identical information as to in the spatial domain [2]. The arrangement of values obtained by applying the DCT is coincidentally from lowest to highest frequency. This characteristic and the psychological observation that the human eye and ear are less sensitive to recognizing the higher-order frequencies leads to the option of compressing a spatial signal by transforming it to the frequency domain and dropping high order values and keeping low-order ones. When reconstructing the data and transforming it back to the spatial domain, the results are remarkably parallel to the original signal. The DCT method can be used to compress both color and gray scale images. DCT is a method most frequently used in several areas including WWW, industries, science and engineering etc [2].

The Haar Wavelet Transformations can be applied only on the NON-ROI parts of the image, where it is used to generate the floating point coefficients. It is a sequence of square-shaped functions but the DCT techniques can also be used for arbitrary shaped functions and zig-zag functions. When DCT technique is used with the JPEG images and the image is reduced to higher compression ratios, the difficulties will raised. So DCT technique with ROI part is used for DICOM images to reduce these difficulties.

In the method, the medical image is selected from the database. Then the image will be segmented into parts and the ROI and NON-ROI part of the image is classified. We will select the ROI part of the image and apply the Discrete Cosine Transform on the image. After applying the selected method both the images are merged together. The quality and size of the original image and newly constructed images are compared [5]. The flow chart of the proposed method is shown below in fig. 1

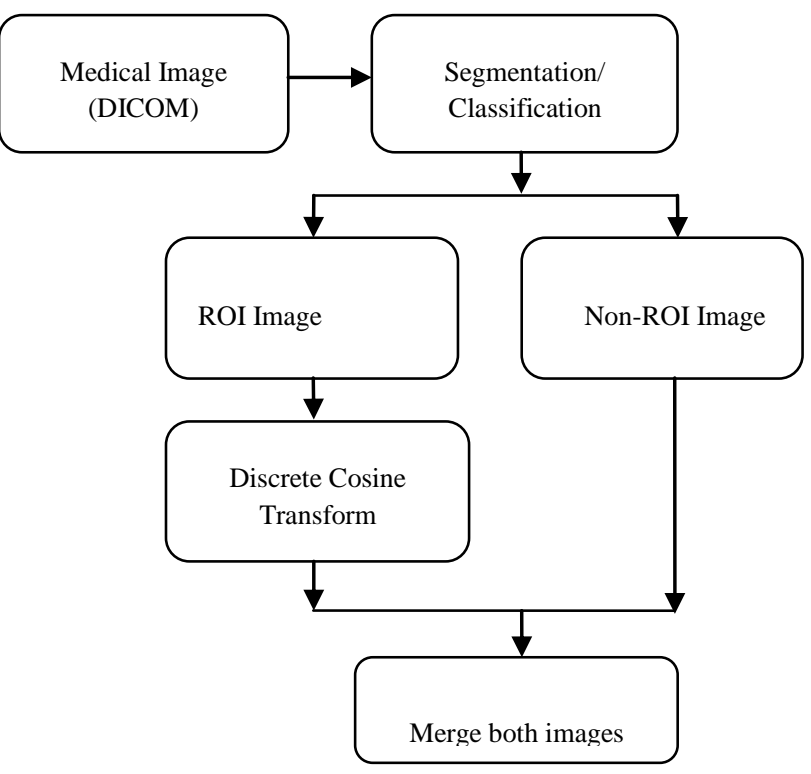

Fig.1 Flow chart of proposed method

\section{CONCLUSION}

The paper discusses about the ROI-based Medical image compression technique. DCT method is used with the ROI part of the image to reduce the blocking effect in the image for the better understanding. Region of Interest based compression techniques helps to reduce size of image without degrading the quality of the important data. The area of improvement can be a system which itself identifies the area of interest within the medical image and then applies various compression techniques on region of interest as well to reduce size of the image.

\section{REFERENCES}

[1]. Gaurav Vijayvargiya, Dr. Sanjay Silakari, Dr. Rajeev Pandey "A Survey: Various Techniques of Image Compression,” International Journal of Computer Science and Information Security (IJCSIS), Issue No. 10, vol. 11, Oct.2013.

[2]. Maneesha Gupta, Dr.Amit Kumar Garg "Analysis Of Image Compression Algorithm Using DCT," International Journal of Engineering Reasearch and Application (IJERA), Website:www .ijera.com, ISSN: 2248-9622, Issue No. 1, Vol. 2, Page No. 515521, Jan-Feb.2012.

[3]. Lavanya. M, M. Suresh Kumar "Intelligent Compression of Medical Images Based on Multi ROI," International Journal of Emerging Technology and advanced engineering (IJETAE), Website: www.ijetae. Com, An ISO 9001:2008 Certified Journal, ISSN: 2250-2459, Issue No.1, Vol.3, Page No.490-493, Jan.2013.

[4]. B. Brindha, G. Raghuraman "Region Based Lossless Compression for Digital Images in Telemedicine Application," IEEE International conference on Communication and Signal Processing, Page No.537-540, India, April 3-5, 2013. 
[5]. Rushab R. Shah, Dr.Priyanka Sharma, Rutvi R. Shah "Performance Analysis of Region Of Interest based Compression Method for Medical Images," IEEE Fourth International Conference on Advanced Computing and Communication Technologies, Page No.53-58, 2014.

[6]. Yung-Gi Wu "Medical Image Compression by Sampling DCT Coefficients," IEEE transactions on information technology in biomedicine, ISSN: 1089-7771, Issue No.1, Page No: 86-94, Vol.6, March.2002.

[7]. V. Radha, Member, International Association of Engineers (IAENG) "A Comparative Study on ROI-Based Lossy Compression Techniques for Compressing Medical Images," Proceedings of the World Congress on Engineering and Computer Science, ISSN: 2078-0966, Vol.1, PageNo.978-988, San Francisco, USA, Oct.19-21, 2011.

[8]. Neelesh Kumar Sahu, Chandrashekhar Kamargaonkar "a survey on various medical image compression techniques," International Journal of Science, Engineering and Technology Research (IJSETR), ISSN: 2278 - 7798, Issue No.2, Vol.2, Page No.501-506, Feb.2013.

[9]. Ilias Maglogiannis, George Kormentzas "Wavelet-Based Compression with ROI coding support for Mobile Access to DICOM image over Hetrogeneous Radio Networks," IEEE transactions on information technology in biomedicine, ISSN:10897771,Issue No.4, Vol. 13, Page No.458-466, July.2009.

[10]. Boqiang Huang, Yuanyuan Wang and Jianhua Chen "2-D Compression of ECG Signals Using ROI Mask and Conditional Entropy Coding," IEEE transactions on biomedical engineering, ISSN: 0018-9294, Issue No.4, Vol. 56, Page No.1261-1263, April.2009.

[11]. Vinayak K Bairagi and Ashok M Sapkal "ROI-based DICOM image compression for telemedicine," Indian Academy of Sciences, Issue No. 1, Vol. 38, Page No.123-131, Feb.2013. 